\title{
THE IMPLEMENTATION OF A RISK STRATIFICATION TOOL FOR THE HAEMATURIA CLINIC TO OPTIMISE THE MANAGEMENT OF PATIENTS WITH HIGH-RISK BLADDER CANCER IN THE COVID-19 ERA
}

Michael Wanis*, Mohammed Kamil Quraishi, Tim Larner

University Hospitals Sussex, Princess Royal Hospital, Haywards Heath, UK

*Author for correspondence: Michael Wanis

E-mail: mikewanis@doctors.org.uk

Submitted: 15 May 2021. Accepted: 29 May 2021. Published 14 July 2021.

\begin{abstract}
Introduction: Elective waiting lists have become more stretched because of the COVID-19 pandemic and patients have evidently been waiting longer for treatment. Patients with high-risk bladder cancer require timely treatment and there is strong evidence to suggest that delay in treatment contributes to a risk of disease progression, metastases and death. Studies have shown that bladder tumour appearances at flexible cystoscopy (FC) can accurately predict high-risk disease on histopathology following transurethral resection. An opportunity for service improvement resulted in a review of the practice followed by the authors and the development of a risk stratification tool for the haematuria clinic which aimed to prioritise the pathway of those with high-risk disease.

Materials and methods: A risk stratification tool was developed for patients with newly diagnosed bladder tumours at the haematuria clinic. A tumour assessment carried out at FC is used to predict patients with high-risk disease, thus allowing those patients to be prioritised over those with low-risk disease on the waiting list. It also includes a reminder to request staging investigations for those with suspected high-risk disease. A closed loop audit was carried to review the following: the quality of tumour risk assessment at the haematuria clinic; time from FC to transurethral resection of bladder tumour (TURBT); concordance between tumour assessment at FC and histopathology after TURBT; efficiency of arranging early staging investigations for those with suspected high-risk bladder cancer; time from FC to staging CT scan.

Results: A risk assessment was carried out for $93 \%$ of patients in the second cycle compared with $40 \%$ in the first cycle. Concordance was noted in $83 \%$ of those with confirmed high-risk non-muscle invasive bladder cancer (NMIBC) and $83 \%$ of muscle invasive bladder cancer (MIBC) in the first cycle, and in 100\% of patients with high-risk NMIBC and MIBC in the second cycle. The interval from FC to TURBT decreased from 27 days in the first cycle to 21 days in the second cycle in those with high-risk NMIBC, and from 27 to 13 days in those with MIBC. Time from FC to staging CT for patients with high-risk bladder cancer was 6 days in the first cycle and 3 days in the second cycle if the request was made from the haematuria clinic. If the CT scan was requested later, the interval increased to 39 days in the first cycle and 22 days in the second cycle.

Conclusion: There is a high degree of concordance between tumour risk assessment at FC and final pathology following TURBT which is supported by several series. Performing risk assessment and requesting staging investigations at the haematuria clinic for patients with newly diagnosed high-risk bladder cancer can minimise delays in their treatment pathway and improve patient outcomes.
\end{abstract}

J Endolum Endourol Vol 4(2):e20-e27; July 14, 2021.

This article is distributed under the terms of the Creative Commons Attribution-

Non Commercial 4.0 International License. (C) Wanis, et al. 


\section{INTRODUCTION}

Bladder cancer is a common disease with an incidence of 10,233 cases and 5485 deaths per year in the UK. $80 \%$ of patients present with NMIBC and $20 \%$ with MIBC. ${ }^{1}$ It is potentially deadly with a $46 \% 10$ year survival overall. ${ }^{2,3}$ The average cost of treatment for patients with NMIBC is $£ 8735$ per patient with additional annual costs of $£ 3957$ and $£ 5407$ to treat disease recurrence and progression, respectively, ${ }^{4}$ and requires significant resources.High-risk non-muscle invasive bladder cancer (NMIBC) and muscle-invasive bladder cancer (MIBC) are associated with an increased risk of disease progression, metastasis and death. Time is of the essence in this patient group, and any delay in treatment could have an adverse effect on oncological outcomes and prognosis. The COVID-19 pandemic has had an adverse impact on elective services in urology, in part due to cancellation of elective surgeries as well as outpatient diagnostic services and clinics. This has reduced the ability to deliver high-quality timely care to cancer patients due to backlogs in waiting lists and resultant delays in referral-to-treatment targets. The Get It Right First Time programme seeks to address long wait times for patients, particularly those on the suspected cancer pathway. ${ }^{5}$ Improving operational efficiency can lead to an increased chance of optimising patient care and meeting performance targets. It has been essential to strike a balance between treating patients with time-critical diseases whilst ensuring adequate resources are available to protect the community from the virus. ${ }^{1,6}$ Anecdotal evidence from the local practice of the authors suggests that patients with newly diagnosed high-risk NMIBC and MIBC are waiting just as long or even longer than some patients with low-risk disease for their primary transurethral resection of bladder tumour (TURBT). Delays have been further exacerbated by limited operating capacity during periods of lockdown.

The COVID-19 pandemic has prompted a review of the practice as well as the introduction of a risk stratification tool for the haematuria clinic to reduce delays and optimise the management of patients with high-risk NMIBC and MIBC with the aim of improving the quality of care that can delivered for this patient group.

\section{METHODOLOGY}

In the first audit cycle, data for all patients with newly diagnosed bladder cancer at the haematuria clinic during the national lockdown periods from March 2020 to May 2020 and November 2020 to January 2021, were collected.We developed a risk stratification tool based on elements of the NICE guidelines ${ }^{7}$ to help triage patients at the haematuria clinic to prioritise patients with suspected high-risk NMIBC and MIBC (Figure 1). This was introduced and disseminated to all members of the team in January 2021. Previously, all patients with cancer or suspected cancer at the haematuria clinic had been assigned "Category 2" on the waiting list request form. "Category 1" procedures are for immediately life-threatening or emergency procedures. By stratifying patients based on risk assessment at flexible cystoscopy (FC), Category 2 was subdivided into Category 2a (suspected high-risk NMIBC and MIBC) and Category $2 b$ (suspected low or intermediate-risk NMIBC).

The risk stratification tool also includes a reminder to request up-front staging investigations for patients suspected to have high-risk NMIBC and MIBC, namely a CT thorax, abdomen and pelvis with a urographic phase to complete their work-up (Figure 1).Data collection included the following information: adequacy and documentation of bladder tumour morphological assessment at $\mathrm{FC}$; concordance between risk assessment at FC and histopathological risk (based on pathological grade and stage) following TURBT; time interval from FC to TURBT; differences in time interval between FC and TURBT between low-risk, high-risk NMIBC and MIBC patients; whether patients with suspected high-risk NMIBC and MIBC had staging investigations requested at the time of $\mathrm{FC}$.

Risk assessment at FC was deemed adequately carried out if the clinician documented features including size, number of tumours (i.e., unifocal or multifocal) and morphological appearance.

A second audit cycle was subsequently carried out from the end of January 2021 to May 2021 following the introduction of our new risk stratification tool to re-evaluate the practice.

In the final analysis, all patients with new bladder tumours diagnosed at the haematuria clinic who

J Endolum Endourol Vol 4(2):e20-e27; July 14, 2021.

This article is distributed under the terms of the Creative Commons Attribution-

Non Commercial 4.0 International License. (c) Wanis, et al. 
subsequently underwent TURBT were included. The following patients were excluded: all patients who were admitted or operated in the emergency setting; patients with a previous diagnosis of bladder cancer; and patients with equivocal findings on $\mathrm{FC}$ or those booked for diagnostic biopsy with an unlikely diagnosis of bladder cancer. Patients whose data were incomplete were also excluded.

\section{RESULTS}

In total, 101 patients underwent TURBT during the first audit cycle and 32 patients in the second audit cycle. 56 patients were excluded from the first cycle and 17 were excluded in the second cycle as they did not meet the inclusion criteria set out previously. Therefore, 45 patients were included in the first cycle and 15 patients in the second cycle. Overall, $77 \%$ of patients were male and $23 \%$ were female, and there was a male preponderance in all three risk groups. Median age was 72 years and was equivocal across all three risk groups.
In the first audit cycle, 9/45 (20\%) patients had low-risk disease $4 / 45 \quad(9 \%)$ patients had intermediate-riskdisease and 32/45 (71\%) had high-risk disease, of which 12/32 had MIBC. The median interval (in days) from FC to primary TURBT for patients with low-risk, intermediate-risk, highrisk NMIBC and MIBC was 25, 27, 27 and 27, respectively (Figure 2).

Patients in the high-risk group waited up to 54 days for their TURBT. Risk assessment at FC was carried out for patients with low-risk, intermediate-risk, high-risk and MIBC in 44, 50, 37 and 48\%, respectively. When risk assessment was carried out for the low-risk, intermediate-risk, high-risk NMIBC and MIBC groups, concordance was noted in 100\% (4/4), $50 \%(2 / 4), 83 \%(10 / 12)$ and $83 \%(5 / 6)$ of patients, respectively (Figure 3).

Staging investigations (i.e., CT thorax, abdomen, and pelvis with urographic phase) were requested for 13/32 (41\%) patients suspected to have high-risk NMIBC or MIBC at FC. The median interval from

\section{Risk stratification of new bladder tumours identified on flexible cystoscopy at Brighton and Sussex haematuria clinic in the COVID era University Hospitals}

If any of the following features are
found, patient is classified as "High
Risk" with Cat 2a on booking form;
patient should also be booked for
CTU and CT chest
- Solid or mixed morphology
appearance of tumour
- Large tumour $>3 \mathrm{~cm}$
- Multifocal tumour

If all of the following are found, patient is classified as "low risk" and booked as Cat 2 on booking form:

- Unifocal tumour

- Papillary

- $</=3 \mathrm{~cm}$

- Non-specific red patch

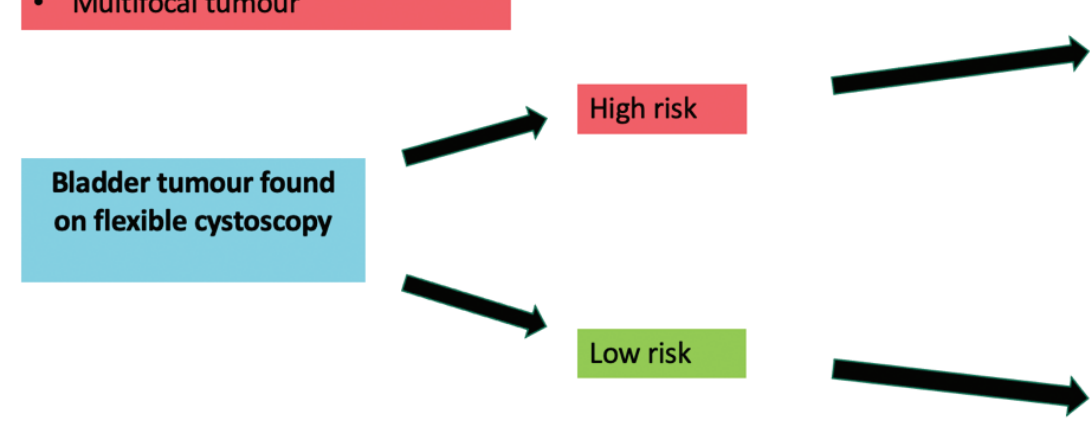

Bladder tumour found on flexible cystoscopy

Figure 1. Risk stratification of new bladder tumours identified on flexible cystoscopy at haematuria clinic in the COVID era. 
$\mathrm{FC}$ to $\mathrm{CT}$ scan was 6 days if the scan was requested at the time of $\mathrm{FC}$ and 39 days if it was not requested at FC (Figure 4). All patients (13/13) completed their staging investigations prior to TURBT if they were requested at the time of $\mathrm{FC}$.

In the second cycle, $2 / 15$ (13\%) patients had lowrisk disease, $3 / 15(20 \%)$ patients had intermediate-risk disease and 9/15 (60\%) patients had high-risk disease, of which 3/9 had MIBC. One patient had benign histology. The median interval (in days) from FC to primary TURBT for patients with low-risk, intermediate-risk, high-risk NMIBC and MIBC was 19, 16, 21 and 13, respectively (Figure 5).

Risk assessment at FC was carried out for patients with low-risk, intermediate-risk, high-risk and MIBC in 100, 100, 89 and $100 \%$ of cases, respectively. When risk assessment was carried out, concordance for the low-risk, intermediate-risk, high-risk NMIBC and MIBC groups, was noted in $100 \%(2 / 2), 33 \%(1 / 3), 100 \%(8 / 8)$ and $100 \%(3 / 3)$ of patients, respectively (Figure 6).

Staging investigations were requested for $7 /$ 9 (78\%) patients suspected to have high-risk NMIBC or MIBC at FC. The median interval from $\mathrm{FC}$ to $\mathrm{CT}$ scan was 3 days if the scan was requested at the time of $\mathrm{FC}$ and 22 days if it was not requested at FC (Figure 7). All patients (7/7) completed their staging investigations prior to TURBT if they were requested at the time of FC.

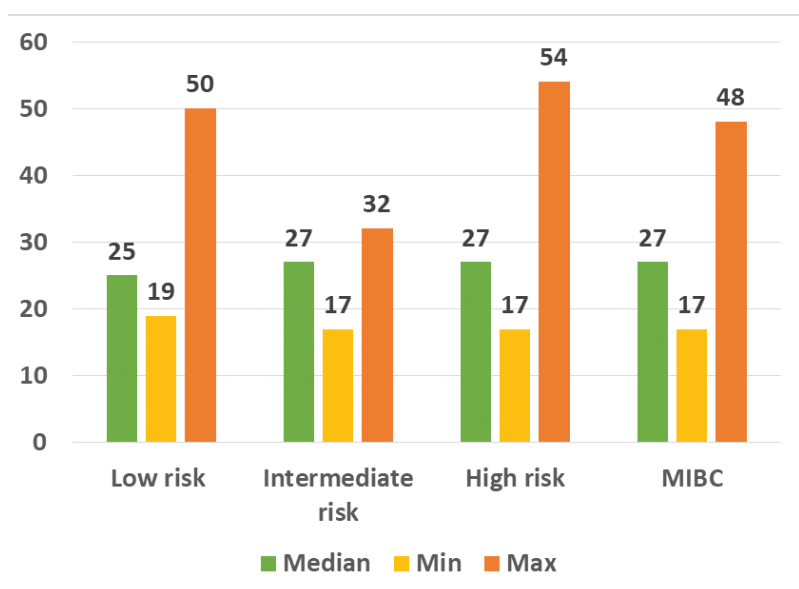

Figure 2. First audit cycle-interval from flexible cystoscopy to TURBT (number of days).

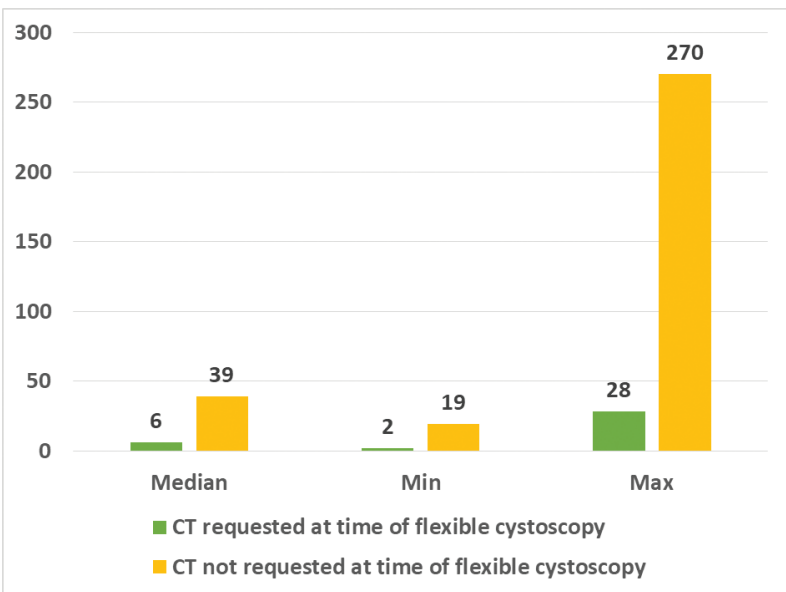

Figure 4. First audit cycle- - time from flexible cystoscopy to staging $\mathrm{CT}$.

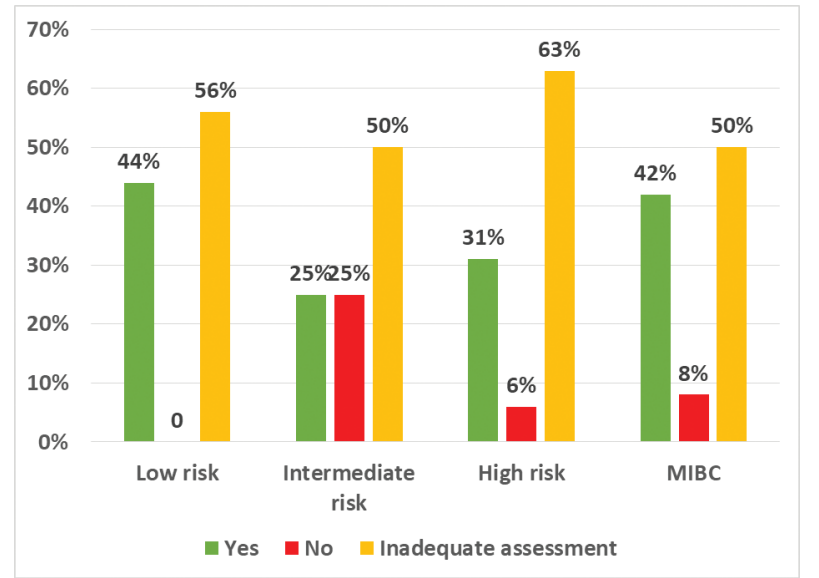

Figure 3. First audit cycle — concordance between risk assessment at flexible cystoscopy and histopathology result following TURBT.

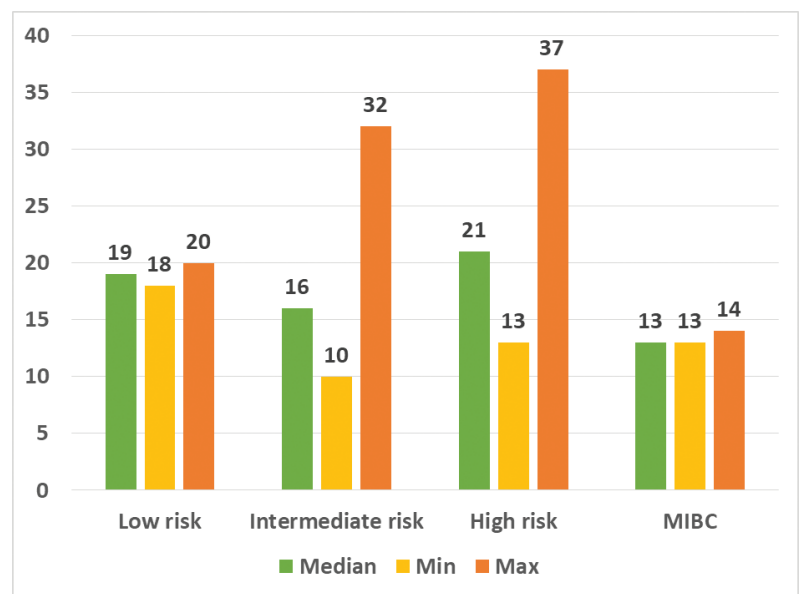

Figure 5. Second audit cycle-interval from flexible cystoscopy to TURBT (number of days).

J Endolum Endourol Vol 4(2):e20-e27; July 14, 2021.

This article is distributed under the terms of the Creative Commons AttributionNon Commercial 4.0 International License. (c) Wanis, et al. 


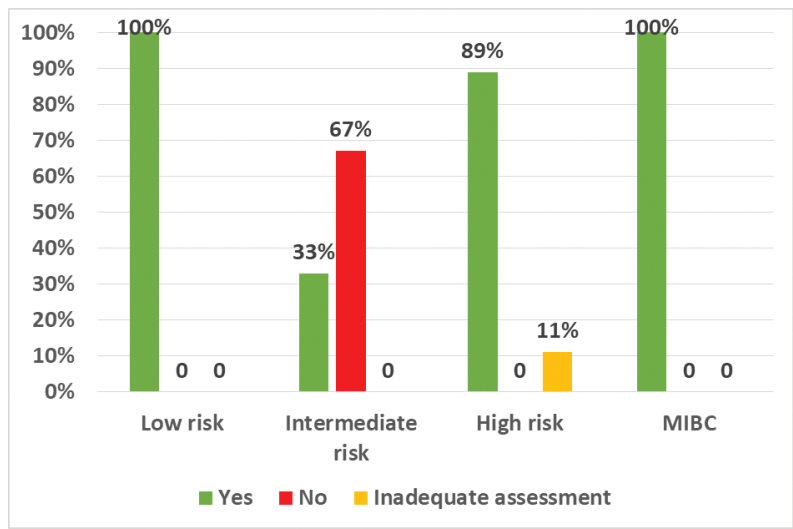

Figure 6. Second audit cycle - concordance between risk assessment at flexible cystoscopy and histopathology results following TURBT.

\section{DISCUSSION}

Although most UK hospitals have resumed normal service with respect to elective surgeries, the aftereffects of the pandemic are likely to be long-lasting and will almost definitely result in added pressures to waiting lists that are already over-stretched. ${ }^{8}$ Patients with high-risk bladder cancer have a long "patient journey" with a pathway encompassing several steps. This complex pathway could lead to delays which may untimely result in treatment for the most high-risk patients. Those with high-risk NMIBC will likely require re-resection at 2-6 weeks followed by initiation of adjuvant intravesical therapy. Those with MIBC will need further discussion at specialist multi-disciplinary team meetings (MDTs), and may require additional workup as well as staging investigations prior to their cystectomy. ${ }^{9}$ This may include cardiopulmonary exercise testing. Some patients will be referred to regional specialist centres for treatment ${ }^{9}$ (Figure 8).

It is also evident that high-risk bladder cancer behaves oncologically different to low-risk disease with different molecular and genetic pathways at play in these distinctly different diseases. ${ }^{10}$ Low-risk NMIBC has a 1 -year and 5 -year risk of progression as low as $<1 \%$. A 3-month delay in treatment is unlikely to lead to adverse outcomes in this group. ${ }^{11,12}$ In the setting of recurrence in this group, there is a role for tumour

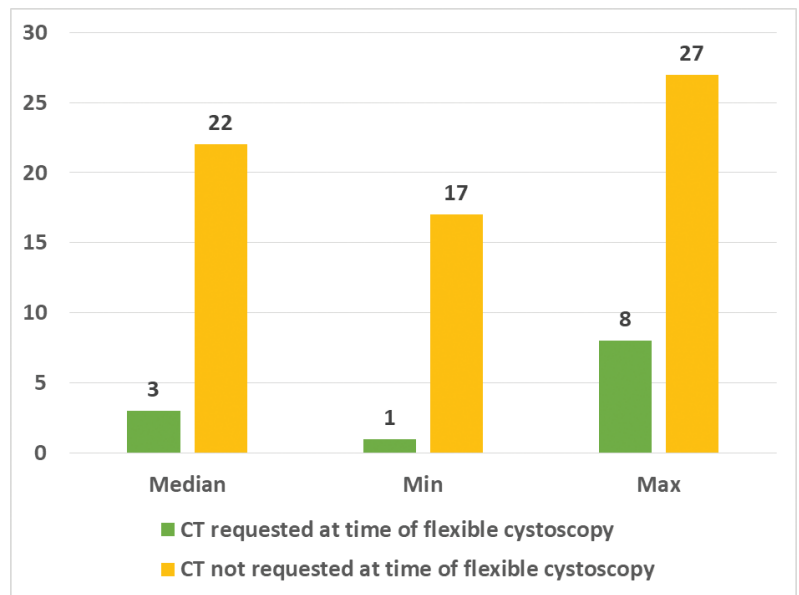

Figure 7. Second audit cycle - time from flexible cystoscopy to staging CT.

fulguration or even active surveillance in some patients, which may be a pertinent option especially in a time of limited resources and capacity. ${ }^{13}$ Conversely, in a study of 198 patients with high-risk NMIBC, up to $25 \%$ of patients progress to MIBC at 5-years despite intravesical BCG therapy. ${ }^{14}$ Further, $48 \%$ of patients with $\mathrm{T} 1$ bladder cancer progress to $\mathrm{T} 2$ or greater if they remain untreated. ${ }^{15,16}$ There is no doubt that MIBC requires prompt and timely treatment, and delay in treatment can result in disease progression and poor outcomes. A study of 126 patients with MIBC who did not receive active treatment revealed a disease-specific mortality of 41,50 and $58 \%$ at 6,9 and 12 months, respectively. Similarly, in a study of 441 patients with MIBC, the risk of death doubled if cystectomy was delayed for 12 weeks compared with patients who underwent cystectomy 4-8 weeks from diagnosis. ${ }^{17}$ A systematic review also confirmed a window of opportunity of 12 weeks from diagnosis to cystectomy in MIBC to avoid metastatic disease. ${ }^{18}$ Several studies have demonstrated that assessment of bladder tumours at FC is able to accurately predict the pathological grade and stage of bladder tumours by taking note of different features including the morphological appearance of the tumour (whether it is papillary, solid or of mixed morphology), size and whether it is unifocal or multifocal. Accuracy rates of up to $91 \%$ for NMIBC and $89 \%$ for MIBC has been demonstrated. ${ }^{19}$ 

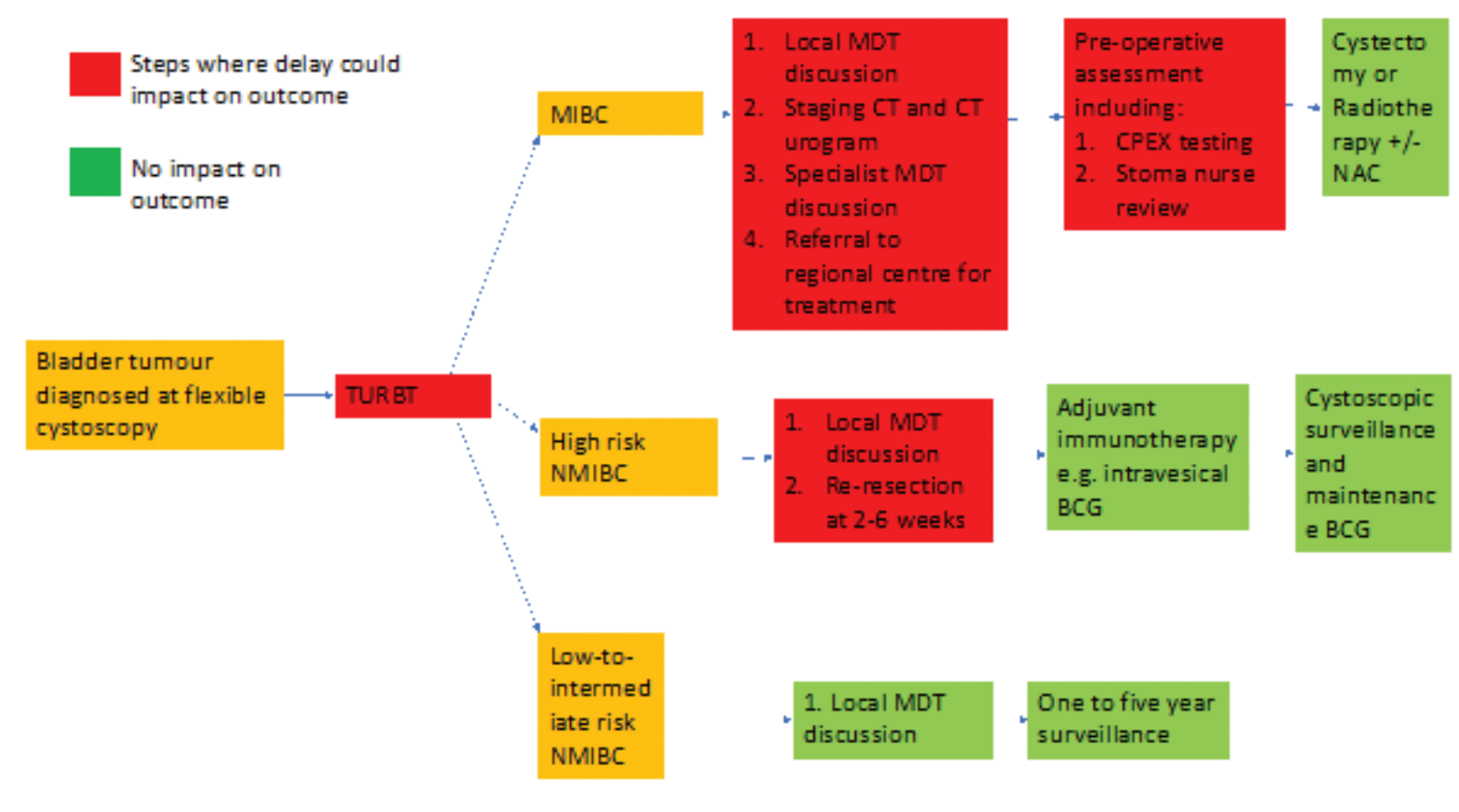

Figure 8.

Studies also show that pathological grading can be predicted with up to $90 \%$ accuracy ${ }^{20}$ and that size and morphological appearance at FC were independent risk factors in predicting muscle invasion. ${ }^{21}$ Tumours that are unifocal, papillary and $<3 \mathrm{~cm}$ are much more likely to represent low-risk NMIBC. ${ }^{20,21}$ This lends itself well to requesting early staging investigations for selected patients at the haematuria clinic. ${ }^{19}$ With the knowledge that significant delays can result in adverse outcomes especially during peak times and pandemic lockdowns when waiting lists are especially overstretched, Evaluation of the practice was with the aim of streamlining the current treatment pathway for patients with newly diagnosed high-risk bladder cancer (including high-risk NMIBC and MIBC). The audit confirmed that patients with high-risk bladder cancer were waiting a median of nearly 4 weeks for their TURBT with some waiting up to 54 days. The interval from FC to TURBT for patients with low-risk NMIBC was almost equivalent to those with high-risk disease. Following the introduction and implementation of the suggested new risk stratification tool at the haematuria clinic, the reaudit showed that this interval was reduced to a median of 21 days (from 27) for

high-risk NMIBC and 13 days for MIBC (from 27). It is interesting to note that the median interval was 19 days for patients with low-risk NMIBC, which is still slightly lower than that for high-risk NMIBC. Although most patients were risk assessed in the second cycle, upon reviewing the waiting list forms, one-third of patients were not subcategorised into $2 \mathrm{a}$ or $2 b$, which could reflect a transition phase in the introduction of the new risk stratification tool. As with most adaptations to clinical pathways, regular education and gentle reminders are needed during this transition phase to optimise the results in the future. The authors are confident that the time interval for the patients with high-risk NMIBC will continue to decrease with time.

In accordance with previously published series, the authors showed that they were able to differentiate between low and high-risk NMIBC as well as MIBC with a good degree of accuracy based on parameters of size, morphological appearance and focality (multifocal or unifocal). Compliance with performing a risk assessment at the time of $\mathrm{FC}$ at the haematuria clinic also improved following introduction of the risk stratification tool, from $40 \%(27 / 45)$ in the first

J Endolum Endourol Vol 4(2):e20-e27; July 14, 2021.

This article is distributed under the terms of the Creative Commons AttributionNon Commercial 4.0 International License. (c) Wanis, et al. 
audit cycle to $93 \%(14 / 15)$ in the second cycle. If a risk assessment was carried out at FC, its accuracy in predicting high-risk NMIBC was $83 \%(10 / 12)$ in the first cycle and $100 \%(8 / 8)$ in the second cycle; and for MIBC was $83 \%(5 / 6)$ in the first cycle and $100 \%$ $(3 / 3)$ in the second cycle.

Moreover, the proportion of patients with suspected high-risk bladder cancer having their staging CT scans requested at the time of $\mathrm{FC}$ increased from $41 \%$ in the first cycle to $78 \%$ in the second cycle. The study data showed that if the staging $\mathrm{CT}$ scan was requested at the time of FC, it was performed in a very timely manner with a median in the first cycle and second cycle of 6 days and 3 days, respectively. However, if it was not requested at the haematuria clinic, the median in the first cycle and second cycle was 39 and 22 days, respectively. This resulted in further delays to the high-risk group.

It was also clear that the staging CT scans were always completed and reported prior to the TURBT if they had been requested at the time of FC. This is a crucial step, both to help inform the surgeon on the day of surgery and to ensure that the full workup is completed in time for MDT. This will avoid further unnecessary delay for patients whilst incurring no additional cost to the department. It is believed that there is further scope for improvement in performance with time and increased compliance of the new stratification tool. Its simplicity also means it is easily transferable and can be utilised in any department to achieve a similar goal.

A limitation of this study includes the relatively small number of cases that were included in the audit. Data from the first audit cycle were collected retrospectively, although the second audit cycle data were prospectively collected. The authors will continue to collect more prospective data and will continue to evaluate their practice with the hope of seeing further improvement in our performance and will endeavour to report on the future results in due course.

\section{CONCLUSION}

To conclude, patients with suspected high-risk NMIBC and MIBC should be prioritised over those with suspected low-risk NMIBC, especially at times when waiting lists are longer than usual. Performing a risk assessment of new bladder tumours at FC can accurately predict patients likely to have high-risk NMIBC or MIBC. Small, unifocal papillary tumours are likely to represent low-risk disease. Our risk stratification tool has enabled us to select out high-risk patients who need urgent, time-critical management, to prioritise them from the start of their treatment pathway with the hope of optimising their outcomes. In addition, a staging CT scan can be requested from the haematuria clinic, allowing a completed workup to be carried out in a timely manner, further minimising any avoidable delay. A team approach with full cooperation and understanding between all staff involved in the haematuria clinic including doctors, nurses, healthcare assistants, administrators, as well as the radiology department is key to ensuring that this endeavour is successful.

\section{ACKNOWLEDGEMENTS}

The authors would like to thank all members of the urology team at University Hospitals Sussex who are involved in the day to day running of the haematuria clinic.

\section{DISCLOSURE}

There is no conflict of interest to declare. No additional funding has been acquired to undertake this work.

\section{REFERENCES}

1. Carvalho F, Galloway L, Saoud R, et al. Considerations about non-metastatic bladder cancer management during the covid-19 pandemic. Bladder Cancer. 2020 May;6(2):99-106. http://dx.doi.org/10.3233/BLC-200311

2. Costing report: Bladder cancer [Internet]. Nice. org.uk. 2015 [cited 2021 May 14]. Available from: https://www.nice.org.uk/guidance/ng2/resources/ costing-report-pdf-3780397

3. Cancer Research UK [Internet]. Cancerresearchuk. org. 2020 [cited 2021 May 10]. Available from: https:// www.cancerresearchuk.org/health-professional/ cancer-statistics/statistics-by-cancer-type/bladdercancer\#: : text $=$ In $\% 20$ females $\% 20$ in $\% 20$ the $\% 20$ UK\%2C\%20bladder\%20cancer\%20is\%20the $\% 20$ 13th,around $\% 203 \% 2 \mathrm{C} 700 \% 20$ deaths $\% 20$ in $\% 202018$

4. Cox E, Saramago P, Kelly J, et al. Effects of bladder cancer on UK healthcare costs and patients' health-related

J Endolum Endourol Vol 4(2):e20-e27; July 14, 2021.

This article is distributed under the terms of the Creative Commons Attribution-

Non Commercial 4.0 International License. (c) Wanis, et al. 
quality of life: Evidence from the BOXIT trial. Clin Genitourin Cancer. 2020 Aug;18(4):418-42. http:// dx.doi.org/10.1016/j.clgc.2019.12.004

5. Harrison S [Internet]. Gettingitrightfirsttime.co.uk. 2018 [cited 2021 May 10]. Available from: https:// www.gettingitrightfirsttime.co.uk/wp-content/uploads/2018/07/Urology-June18-M.pdf

6. Wallis C, Novara G, Marandino L, et al. Risks from deferring treatment for genitourinary cancers: A collaborative review to aid triage and management during the COVID-19 pandemic. Eur Urol. 2020 July;78(1):29-42. http://dx.doi.org/10.1016/j.eururo.2020.04.063

7. NICE guidelines [Internet]. Nice.org.uk. 2015 [cited 2021 May 10]. Available from: https:// www.nice.org.uk/guidance/ng2/resources/ bladder-cancer-diagnosis-and-management-pdf-51036766405

8. The Lancet Rheumatology. Too long to wait: The impact of COVID-19 on elective surgery. Lancet Rheumatol. 2021 Feb;3(2). http://dx.doi.org/10.1016/ S2665-9913(21)00001-1

9. Babjuk M, Burger M, Comperat E, et al. uroweb. org. [Internet]. Uroweb.org. 2021 [cited 2021 May 10]. Available from: https://uroweb.org/guideline/ non-muscle-invasive-bladder-cancer/

10. Knowles M, Hurst C. Molecular biology of bladder cancer: New insights into pathogenesis and clinical diversity. Nat Rev Cancer. 2015 Jan;15(1):25-41. http://dx.doi.org/10.1038/nrc3817

11. Sylvester R, Oosterlinck W, Holmang S, et al. Systematic review and individual patient data meta-analysis of randomized trials comparing a single immediate instillation of chemotherapy after transurethral resection with transurethral resection alone in patients with stage pTa-pT1 urothelial carcinoma. Eur Urol. 2016 Feb;69(2):231-44.

12. Wallis C, Catto J, Finelli A, et al. The impact of the COVID-19 pandemic on genitourinary cancer: Reenvisioning the future. Eur Urol. 2020 Nov;78(5):731-42. http://dx.doi.org/10.1016/j.eururo.2020.08.030
13. Matulay J, Soloway M, Witjes J, et al. Risk-adapted management of low-grade bladder tumours: Recommendations from the international bladder cancer group (IBCG). BJUI. 2020 Jan;125(4):497-505. http:// dx.doi.org/10.1111/bju.14995

14. Herr H. Tumor progression and survival of patients with high grade, noninvasive papillary (TaG3) bladder tumors: 15-year outcome. J Urol. 2020 Jan;163(1):60-1. http://dx.doi.org/10.1016/S0022-5347(05)67972-4

15. Nieder A, Brausi M, Lamm D, et al. Management of stage $\mathrm{T} 1$ tumors of the bladder: International consensus panel. Urology. 2005 Dec;66(6):108-25. http://dx.doi. org/10.1016/j.urology.2005.08.066

16. Pasin E, Josephson D, Mitra A, et al. Superficial bladder cancer: An update on etiology, molecular development, classification, and natural history. Rev Urol. 2008;10(1):31-43.

17. Gore J, Lai J, Setodji C, et al. Mortality increases when radical cystectomy is delayed more than 12 weeks. Cancer. 2009 Jan;115(5):988-96. http://dx.doi. org/10.1002/cncr.24052

18. Fahmy N, Mahmud S, Aprikian A. Delay in the surgical treatment of bladder cancer and survival: Systematic review of the literature. Eur Urol. 2006 Dec;50(6):1176-82. http://dx.doi.org/10.1016/j. eururo.2006.05.046

19. During V, Sole G, Jha A, et al. Prediction of histological stage based on cystoscopic appearances of newly diagnosed bladder tumours. Ann R Coll Surg Engl. 2016 Nov;98(8):547-51. http://dx.doi.org/10.1308/ rcsann.2016.0246

20. Mitropoulos D, Kiroudi-Voulgari A, Nikolopoulos P, et al. Accuracy of cystoscopy in predicting histologic features of bladder lesions. J Endourol. 2005 Sept;19(7):861-4. http://dx.doi.org/10.1089/end.2005.19.861

21. Satoh E, Miyao N, Tachiki H, et al. Prediction of muscle invasion of bladder cancer by cystoscopy. Eur Urol. 2002 Feb;41(2):178-81. http://dx.doi.org/10.1016/ S0302-2838(01)00035-5

J Endolum Endourol Vol 4(2):e20-e27; July 14, 2021.

This article is distributed under the terms of the Creative Commons AttributionNon Commercial 4.0 International License. (c) Wanis, et al. 